\title{
Summary of the Special Issue "Numerical Analysis, Material Modeling and Validation for Magnetic Losses in Electromagnetic Devices"
}

\author{
Zhiguang Cheng \\ Institute of Power Transmission and Transformation Technology, Baobian Electric Co., Ltd, Baoding, China
}

Email address:

emlabzcheng@yahoo.com

\section{To cite this article:}

Zhiguang Cheng. Summary of the Special Issue. International Journal of Energy and Power Engineering. Special Issue: Numerical Analysis, Material Modeling and Validation for Magnetic Losses in Electromagnetic Devices. Vol. 5, No. 1-1, 2016, pp. 75-77.

doi: $10.11648 /$ j.ijepe.s.2016050101.21

\section{Academic and Industrial Background}

\subsection{New Challenges in Electromagnetic Analysis and Design}

Along with the requirement to significantly increase the voltage and the capacity of the power transmission and transformation equipment, to the highest level in the history of the world, came the requirement for much more efficient computer-aided analysis and design techniques, involving a more accurate modeling of the material properties. In other words, when simulating a low voltage/capacity equipment, some errors in the approximations, used in the numerical techniques, are not as critical, but must be considered for the case of the high voltage and large capacity equipment.

\subsection{Combination of Material Modeling and Numerical Analysis}

It should be noted that the research and development of advanced material modeling techniques, at the present, is not fully integrated in the computer aided simulations. The application of advanced material property modeling techniques, in combination with an efficient numerical analysis system, is very important, relevant not only to material modeling and measurement methods, but is also relevant to materials manufacturers, power transmission and transformation equipment manufacturers, requiring consensus from the scientific community, and cooperation between the industrial and scientific communities.

\subsection{Modeling of Working Magnetic Material Properties}

The magnetic properties of materials used in industrial applications are usually measured under specified standard conditions, which may be considerably different from the real working conditions of the material used in the components of a device in operation. Under some extreme running conditions, the model may even require a multi-physics coupled field solution (e.g., conditions including harmonic and/or DC-biased excitations). For an accurate simulation, such working material property data are needed and should be supported in the simulation code.

\subsection{Improvement and Application of Material Modeling Techniques}

Even though the classical measurement methods for the magnetic material properties have been used for many years, there are some problems worthy of further research including comparative studies among different measurement methods, which could lead to improvements in dealing with the effects of some engineering factors on the widely used property data.

\subsection{Validation and Effectiveness of Modeling and Numerical Simulations}

Today, the effectiveness and usefulness of modeling and simulation, under extreme excitations, is even more significant, and at the same time, the validation of the simulation results has become very challenging. Here, modeling has a number of facets, 
including academic and engineering-based simplification, the numerical technique used, sufficient property data, efficient computation methods and well-established experimental techniques.

\section{Outline of the Special Issue}

The accurate computation of magnetic losses in large electromagnetic devices, possibly under extreme excitations, has become increasingly important and remains to be very challenging, requiring an efficient numerical field solution and a magnetic material model that simulates the true material behavior, as experienced when the equipment is under operating conditions; the latter requirement necessitates a validation procedure that is based on the characteristics of the equipment itself. This special issue selectively presents the related co-research works undertaken by our colleagues from the university, the research institute and the industry.

\subsection{Efficient Solution of Large-Scale Electromagnetic Fields}

The finite element solution of the electromagnetic fields in some electrical machines and transformers poses a number of problems, such as large scale computation, resulting in very long solution times and poor precision which could not meet the demands of engineering accuracy. An overview of the current research situation and the difficulties of these problems are given mainly from the aspect of computation methodology. The methods to deal with these problems, e.g., homogenization models of the laminated iron core, the sub-domain perturbation finite element method, the domain decomposition method, and EBE (Element by Element) parallel finite element method, are described. Their advantages and limitations are discussed, and the authors' suggestions for the further research strategies are also included. See "Finding better solutions to reduce computational effort of large-scale engineering eddy current fields" by D. Xie et al.

\subsection{Developments in Advanced Numerical Analysis and Design Methods}

A parallel finite element toolbox for computing fields in large electromagnetic devices, using unstructured tetrahedral meshes, FEMAG-Fem for ElectroMagnetics on Adaptive Grids, is presented. The finite element toolbox deals with unstructured tetrahedral meshes and can solve electromagnetic eddy current problems in both the frequency domain and time domain. It adopts high-order edge element methods and refines the mesh adaptively based on reliable and efficient finite element a posteriori error estimates. The competitive performance of FEMAG is demonstrated through extensive numerical experiments, including TEAM (Testing Electromagnetic Analysis Methods) Problem 21 and the simulation of a single-phase power transformer. See "FEMAG: a high performance parallel finite element toolbox for electromagnetic computations", by T. Cui, W. Zheng, et al.

A new method, to optimally determine the fixed-point reluctivity, is presented that ensures a stable and fast convergence of harmonic solutions. The nonlinear system matrix is linearized by using the fixed-point technique, and harmonic solutions are decoupled by the diagonal reluctivity matrix. The 1-D and 2-D non-linear eddy current problems under DC-biased magnetization are computed by the proposed method. The computational performance of the new algorithm proves its validity and efficiency. The corresponding decomposed method is proposed to solve the nonlinear differential equation, in which the harmonic solutions of the magnetic field and the exciting current are decoupled in the harmonic domain. See "Fixed-point harmonic-balanced method for nonlinear eddy current problems" by X. Zhao et al.

The harmonic-balanced method can be used to compute the time-periodic electromagnetic field in the harmonic domain, considering electric circuits coupled with the nonlinear magnetic field. 2-D and simplified 3-D model of the laminated core is developed and is used in the computation, proving the accuracy and validity of the proposed method. The calculated magnetizing current is compared with the measured results and the computed magnetic field is analyzed to investigate the effect of DC bias. See "Computation and Analysis of the DC-biasing magnetic field by the harmonic-balanced finite-element method" by X. Zhao et al.

The magnetization and loss properties are analyzed and compared for topical magnetic materials. The calculation methods are studied for the losses of the core and the windings of a High Frequency Transformer (HFT). Based on the evaluation of the temperature increase, an optimization method is presented for the design of HFT. Finally, the losses of a test model of HFT is calculated and the results are compared with those tested. See "Loss calculation and optimization design of high frequency transformer" by L. Li et al.

\subsection{Magnetic Property Modeling and Numerical Simulation}

The modeling and numerical analysis of the magnetic loss inside the components of large and special electromagnetic devices, excited by sources containing multiple harmonics, with or without a DC bias, are increasingly of interest. The goal of this paper is to investigate efficient and reliable approaches that can be used to determine the magnetic losses inside both the solid and laminated components under such extreme excitations. All the proposed approaches are experimentally validated. See "Magnetic loss inside solid and laminated components under extreme excitations" by Z. Cheng, B. Forghani, et al. 
The leakage flux and the iron loss generated in the laminated silicon sheets of the core and the magnetic shields of large power transformers are evaluated. A verification model is established, and the parabolic model (non-saturated regions) and the hybrid model (saturation regions) are proposed to simulate the magnetic properties of the silicon steel with different angles to the rolling direction. An efficient analysis method is implemented and validated. The calculated and measured results with respect to the test models are in good agreement. See "Modeling and analysis of leakage flux and iron loss inside silicon steel laminations" by Y. Du et al.

The noise and vibration problems in power transmission and transformation systems are increasingly of concern, requiring a systematic and fundamental research on the magnetostriction property of silicon steel sheets. The measurement method, the property model formulation, and the application of the model to finite element analysis are described. The relative numerical results are also presented. See "Research on magnetostriction property of silicon steel sheets" by Y. Zhang, D. Xie, et al.

The magnetic properties of grain oriented electrical steel, under the controllable distorted flux condition, is investigated, based on a product-level core model. This work examines the effects of the distorted flux on the magnetic loss inside the laminated cores using different excitation conditions, involving different harmonic phase difference, harmonic order and harmonic contents. See "Effect of controllable distorted flux on magnetic loss inside laminated core" by Y. Liu et al.

\subsection{Engineering-Oriented Validation}

There are two important aspects of the electromagnetic field modeling and simulation when used for industrial applications, namely the application-based benchmarking activities and the magnetic material modeling. This work emphasizes the relationship between the two, and briefly reviews the recent progress in extending the TEAM (Testing Electromagnetic Analysis Methods) Problem 21 Family (P21) and the related modeling results, and proposes a new benchmarking project which includes the upgraded benchmark models that can handle extreme excitations, i.e. current sources with a DC bias, as well as multiple harmonics. See "Extended P21-based benchmarking" by Z. Cheng, B. Forghani, et al.

Finally, as I have stated above, further study on the topics discussed in this special issue have been undertaken by our teams of researchers which will lead to new and improved methodologies and results; those will be reported elsewhere. I really appreciate all the comments, suggestions and advice received from all the colleagues who took interest in the research works presented in this special issue.

\section{Acknowledgement}

I would like to express my gratitude to all the colleagues for their cooperation for many years, for unselfishly sharing their time to complete the manuscripts, and enthusiastically making this special issue valuable. Meanwhile, some further co-research works are undertaken as new proposed projects.

This project was supported in part by the State Grid Corporation of China under Grants sgri-wd-71-13-002, by Baobian Electric Co., Ltd, under grant JC11, and by the Foundations under Grants, as stated in the corresponding contribution papers of this Special Issue.

I would like to specially thank the team at Infolytica, Canada, for their co-research and support. 